Maria Elias Abadias*; David Chesa**; Eulalia Izquierdo***;

Eduard Fernández****; Montserrat Sitjas *****

\title{
Eficacia de la rehabilitación cognitiva en la esquizofrenia: una revisión
}

RESUMEN: En las últimas décadas ha habido un aumento importante del numero de estudios centrados en los aspectos neuropsicológicos de la esquizofrenia, los cuales han pretendido conocer los déficits cognitivos que presentan los pacientes esquizofrénicos y las consecuencias de estos déficits en su adaptación funcional. El reconocimiento de la importancia de los déficits cognitivos en la esquizofrenia ha generado un creciente interés por la posible modificación de éstos a través de la rehabilitación cognitiva. En este trabajo presentamos los principales déficits neuropsicológicos asociados a la esquizofrenia y las consecuencias de estos déficits y realizamos una revisión en relación a los efectos de la rehabilitación cognitiva en el rendimiento cognitivo y social de las personas con esquizofrenia.

Palabras clave: Esquizofrenia. Rehabilitación cognitiva. Déficit cognitivo. Déficit funcional. Eficacia.
SUMMARY: The number of studies about the neuropsichology of the schizophrenia has grown up in the last decades. These studies have tried to know the cognitive deficits that show the schizophrenic patients and the consecuences of these deficits in their adaptation. The increasing awareness of the importance of the neurocognitive impairments has fostered considerable interest in the prospects for cognitive rehabilitation. In this paper we present the main cognitive deficits on schizophrenia and their consecuences and we review the impact of cognitive rehabilitation in cognitive and social outcome for individuals with schizophrenia.

Key words: Schizophrenia. Cognitive rehabilitation. Cognitive deficit. Functional deficit. Eficacy. 


\section{Iintroducción}

El trastorno esquizofrénico cursa con una elevada frecuencia de estados o síntomas deficitarios después de la desaparición de la sintomatología aguda. Entre un 50 y $80 \%$ de las personas con esquizofrenia presenta rendimientos neuropsicológicos dentro del rango de pacientes orgánicos cerebrales $(1,2,3)$. Este déficit puede dificultar la adaptación psicosocial del paciente. Es a partir de estos aspectos que se plantea la necesidad de la rehabilitación cognitiva en pacientes esquizofrénicos.

Las preguntas que surgen a raíz de la puesta en marcha de los programas de rehabilitación cognitiva son las siguientes: ¿Es posible rehabilitar el déficit cognitivo en pacientes con esquizofrenia? Y en segundo lugar, ¿Se generalizan los resultados del entrenamiento a la vida cotidiana?

Si nos fijamos en los antecedentes históricos, el bajo rendimiento en los tests neuropsicológicos de los pacientes con esquizofrenia ha sido documentado principalmente los últimos 30 años. Sin embargo, en 1919 Kraepelin ya apuntaba la dificultad que presentaban los pacientes esquizofrénicos para mantener la atención centrada en una tarea. Bleuler en 1911 indica como síntoma fundamental de la esquizofrenia el trastorno asociativo.

Los primeros ensayos de aplicación de programas de rehabilitación neuropsicológica los hallamos a principios de los años 70 con autores como Wagner, Meichenbaum, Platt y Spivack. Estos estudios no fueron replicados y el interés por la rehabilitación cognitiva se fue debilitando. Fue a finales de los años 80 cuando se reaviva el interés por la rehabilitación cognitiva. Esto es debido a la aparición de importantes estudios que evidenciaban la presencia de déficits cognitivos en la esquizofrenia los cuales eran considerados un elemento central del trastorno.

\section{Déficit cognitivo en la esquizofrenia}

a) Características del déficit cognitivo

El déficit cognitivo que se presenta en los pacientes esquizofrénicos puede ser de distintos tipos. Aún así aparecen unas características comunes:

- Los patrones de disfunción están relacionados de forma primaria y patofisiológicamente con el trastorno, y no son una consecuencia de la cronicidad, el tratamiento o la institucionalización(4,5).

- El déficit neuropsicológico no se relaciona con la edad actual, ni con la edad de inicio del trastorno ni con la duración de éste (6).

- Se plantea la esquizofrenia como una encefalopatía estable, más que como un proceso degenerativo, en la cual las disfunciones cognitivas se estabilizan los primeros 4 o 5 años del trastorno(6,7,8,9,10).

- El déficit neuropsicológico es superior en pacientes con sintomatología negativa (11). 
- Una proporción significativa de pacientes jóvenes, agudos y de primer episodio, presenta un deterioramiento en el CI respeto al nivel intelectual premórbido(12,13).

- Según los modelos cognitivos de la esquizofrenia las alteraciones neuropsicológicas son la expresión de un mal funcionamiento del cerebro debido probablemente a una infraactivación metabólica del lóbulo frontal y a anormalidades en los circuitos neuronales que unen los lóbulos frontal, temporal y límbico(14). El déficit se situaría a nivel cortical y subcortical con predominio de circuitos frontotemporales.

Con relación a las alteraciones neuropsicológicas específicas, éstas se detectan principalmente en las funciones ejecutivas, la memoria y la atención.

- En las funciones ejecutivas se obtienen rendimientos significativamente inferiores que en sujetos normales. Las áreas más afectadas de las funciones ejecutivas son: la fluidez verbal, la categorización y la flexibilidad cognitiva(15).

- En la memoria se han detectado déficits en distintos aspectos mnésicos: peor rendimiento en tareas de memoria reciente seguidas de las de memoria a corto plazo y de codificación(16) y déficit en la memoria de trabajo $(10,17)$.

- En cuanto a la atención, prerrequisito general y necesario del funcionamiento cognitivo, el aspecto más afectado es la atención sostenida(18). Este déficit está presente en el período premórbido, en la fase aguda y en la remisión(15).

b) Trastorno cognitivo generalizado vs. focal

Se ha planteado la cuestión de si los pacientes con esquizofrenia presentan un trastorno neuropsicológico generalizado o focal. Los resultados obtenidos en los distintos estudios apoyan la hipótesis de la existencia de déficits cognitivos diferenciales que coexistirían con un déficit cognitivo generalizado(3,19). Aún así, Rund y Borg(15) consideran que la ausencia de estudios metodológicamente sólidos impiden establecer una conclusión firme respeto al déficit cognitivo selectivo

c) Subtipos de déficit cognitivo

Debido a la heterogeneidad del deterioro cognitivo en la esquizofrenia, Heinrichs y Awas intentan establecer subtipos de esquizofrenias en función de la estructura cerebral implicada y el déficit neuropsicológico asociado:

1. Córtex prefrontal: subtipo prefrontal de esquizofrenia que se caracteritzaría por un deterioro de las funciones ejecutivas con una relativa preservación de las otras funciones neurocognitivas.

2. Hipocampo y tálamo medial: subtipo hipocámpico-diencefálico relacionado con una disfunción mnésica que se caracterizaría por un rápido olvido de información recientemente aprendida y recuerdo contaminado con material irrelevante.

3. Ganglios basales: alteraciones que afectarían a la conducta y a la planificación motora. 
4. Disfunción cerebral difusa: alteraciones cognitivas generalizadas que se caracterizarían por la existencia de un déficit difuso en varias áreas cerebrales conectadas, como por ejemplo los circuitos córtico-subcorticales(20,21). Estos déficits pueden estar ya presentes antes del inicio del trastorno clínico(22).

5. Es en este sentido que actualmente muchos autores creen que el modelo clásico basado en déficits segregados es inadecuado en la esquizofrenia y plantean un modelo derivado de los conceptos de integración neuronal. Nos hallamos ante las llamadas redes cerebrales a gran escala(23).

\section{Consecuencias del dèficit neuropsicològico}

Se ha estudiado el papel que juegan los déficits cognitivos con relación al trastorno esquizofrénico y se ha visto que tienen efectos sobre distintos aspectos:

1. Adaptación funcional

El déficit neuropsicológico se ha relacionado con peor rendimiento en el funcionamiento social, el trabajo y el grado de autonomía $(24,25,26,27,28,29)$. Green(30) lleva a cabo una revisión analizando los estudios empíricos recientes más relevantes y concluye que:

- Las funciones ejecutivas están fuertemente asociadas al funcionamiento en la comunidad.

- La memoria verbal tiene un papel importante en todos los tipos de rendimiento psicosocial.

- La vigilancia está relacionada con la solución de problemas sociales y la adquisición de habilidades.

2. Curso de la Esquizofrenia

Se obtienen importantes correlaciones entre el déficit neuropsicológico y el curso de la esquizofrenia $(31,32,33)$, en el sentido que en pacientes con mayor déficit cognitivo se detecta un peor curso del trastorno.

3. Intervenciones psicosociales

En varios estudios se ha observado que el déficit cognitivo interfiere en las intervenciones psicosociales tales como el entrenamiento en habilidades y la terapia cognitivoconductual $(34,35,36)$.

A partir de estos datos vemos que el déficit cognitivo puede actuar como factor limitador, impidiendo a los pacientes alcanzar una buena adaptación funcional. Este hecho plantea la necesidad de una intervención a nivel neuropsicológico.

\section{3. ¿Se puede modificar el déficit neuropsicológico?}

Al plantearnos la intervención en el déficit neuropsicológico, lo primero que debemos preguntarnos es si es posible modificar este déficit en los pacientes esquizofrénicos. Para 
conocer este aspecto se han realizado diferentes estudios experimentales centrados en el entrenamiento de funciones específicas. Estos programas específicos los podemos agrupar en habilidades conceptuales (funciones ejecutivas), atencionales y mnésicas.

a) Funciones ejecutivas

El paradigma experimental más utilizado para estudiar si se puede modificar el déficit en las funciones ejecutivas en pacientes esquizofrénicos ha sido el entrenamiento en el test de WCST (Wisconsin Card Sorting Test). Se trata de un índice de funcionamiento prefrontal en el cual el $80 \%$ de los pacientes esquizofrénicos obtienen puntuaciones inferiores a los sujetos normales(20,37). En esta prueba se pide al sujeto que realice una clasificación de tarjetas según distintas categorías (color, forma y número). El evaluador cambia el criterio de clasificación sin advertir al sujeto, informándolo únicamente de si la clasificación ha sido correcta o no.

Los primeros estudios con el WCST son los de Goldberg y col.(38). Estos crean un paradigma experimental utilizando el WCST en el cual entrenan a los sujetos para realizar correctamente el test. Obtienen ausencia de aprendizaje en todas las condiciones experimentales y concluyen que el déficit cognitivo es inmodificable.

Posteriormente se presentan una serie de estudios que contradicen los resultados obtenidos por estos autores:

Green y col.(39) realizan un estudio para comprobar si modificando el procedimiento de entrenamiento en pacientes con importantes déficits cognitivos podían mejorar su rendimiento en el WCST. Se administra el test a 46 pacientes esquizofrénicos crónicos y 20 sujetos control con otras enfermedades psiquiátricas. Todos los sujetos pasan por cuatro condiciones experimentales que varían en el refuerzo monetario y el nivel de instrucciones.

En los resultados obtenidos se evidencia que el refuerzo monetario tiene cierto efecto en la ejecución del test. Pero el hecho de dar instrucciones de forma detallada mejoraba significativamente las puntuaciones tanto en el número de respuestas correctas, como en el número de errores perseverativos y el de categorías completadas.

Estos resultados son replicados en un gran número de investigaciones. En éstas se muestran los efectos positivos del entrenamiento en el WCST, especialmente cuando se proporciona un refuerzo positivo a las respuestas correctas y se facilita el uso de auto instrucciones y guías internas $(40,41,42,43,44,45,46,47,48)$.

Consecuentemente, se pone de relieve que un breve entrenamiento mejora el rendimiento de la función ejecutiva en el test. Aún así no hay evidencia que este aumento en el rendimiento en el test se equipare a una mejora de la función cognitiva(49).

b) Atención

En el ámbito experimental, para estudiar la posibilidad de modificar el déficit en la atención en pacientes con esquizofrenia, se han utilizado pruebas neuropsicológicas como son el CPT, el SPAN y los listados de repetición de palabras. 
Los principales estudios realizados obtienen una mejora en la ejecución en los tests en los cuales los sujetos habían sido entrenados. Afirmando así los autores que la práctica en una tarea de atención puede mejorar la ejecución en esa tarea específica. No obstante consideran que hay poca evidencia con relación a la generalización de este entrenamiento(50).

Suslow y col. (51) realizan una revisión de los efectos del entrenamiento cognitivo de la atención en pacientes esquizofrénicos. Recogen los nueve estudios que consideran metodológicamente más adecuados y obtienen que proporcionan resultados inconsistentes entre ellos. Sus análisis les conducen a establecer que, con relación a la efectividad del entrenamiento cognitivo en atención, la evidencia aún no es concluyente, siendo necesario realizar estudios en los cuales se sistematicen los aspectos de la atención a evaluar y entrenar.

c) Memoria

En lo referente a la rehabilitación del déficit en memoria, nos hallamos en el campo en el cual ha habido menos intentos de abordaje específico. Uno de los principales estudios es el de Koh y col. $(52,53)$ donde muestran resultados positivos en una tarea de memoria cuando la codificación era ayudada mediante la clasificación de estímulos.

d) Rehabilitación cognitiva y pruebas de neuroimagen

Utilizando pruebas de neuroimagen se ha pretendido conocer el efecto de la rehabilitación cognitiva a nivel del SNC. Hasta el momento los estudios realizados con pruebas de neuroimagen se basaban en muestras reducidas de sujetos y sin grupo control. Los resultados de estos trabajos ya apuntaban a un cambio en la activación en determinadas áreas del SNC de forma posterior a la participación de los sujetos en los entrenamientos cognitivos $(54,55)$.

Posteriormente Wykes y col.(56) publicaron un estudio que cumple los requisitos metodológicos. En este se obtiene que en los pacientes que han participado con éxito en la CRT (Cognitive remediation therapy) se da un incremento significativo de la activación cerebral en regiones asociadas a la memoria de trabajo, particularmente en áreas frontocorticales. Siendo este el primer estudio en el que se pueden asociar los cambios en la activación cerebral a una intervención psicológica en vez de farmacológica.

e) Conclusiones en relacion a la modificabilidad del déficit cognitivo específico Analizando los resultados de todos estos trabajos sobre la rehabilitación del déficit cognitivo específico hallamos que han demostrado que la ejecución en determinados tests neuropsicológicos puede ser mejorada mediante el entrenamiento. Aún así, estas mejoras parecen quedar restringidas al procesamiento elemental de información. No hay evidencia concluyente con relación a como estas mejoras en los tests 
neuropsicológicos se generalizan a funciones cognitivas más complejas, habilidades sociales y adaptación(57).

Otro aspecto importante a tener en cuenta a la hora de valorar los resultados de los estudios son ciertas insuficiencias metodológicas que presentan. La mayoría de estos estudios se realizan con muestras muy reducidas, sin grupo control y con un tiempo corto de observación que no permite conocer la estabilidad de estas mejorías.

\section{Eficacia de los programas de rehabilitación cognitiva}

Una vez se pudo objetivar la posibilidad de modificar el déficit cognitivo de los pacientes con esquizofrenia, se desarrollaron una serie de programas para aplicar estos hallazgos experimentales al ámbito clínico. A continuación presentamos los resultados obtenidos con relación a la eficacia rehabilitadora de los distintos programas de intervención.

\section{A. Terapia psicológica integrada}

Uno de los programas más relevantes y que ha generado un mayor numero de investigaciones es la Terapia Psicológica Integrada de Brenner (IPT)(58).

Se trata de un programa de orientación conductual diseñado para mejorar las habilidades cognitivas y sociales de pacientes esquizofrénicos. Se fundamenta en la Teoría de la Vulnerabilidad de Zubin y Spring(59) según la cual varios factores etiológicos llegarían a provocar una vía intermedia común que es la vulnerabilidad. Está compuesto por 5 módulos orientados a paliar el déficit cognitivo y a la vez mejorar el comportamiento social deficitario.

Se han realizado un gran número de estudios para conocer su eficacia. Los autores del IPT han realizado más de veinte estudios con setecientos pacientes de distintos países y obtienen rendimientos significativamente mejores en los tests de atención, formación de conceptos y pensamiento abstracto(60).

El mejor estudio publicado hasta la actualidad, debido a su pureza metodológica, es el de Spaulding y col.(61). Evalúan los efectos del IPT aplicado durante seis meses a noventa pacientes de media estancia. Todos los pacientes participan en un programa de rehabilitación global (que incluye, entre otras intervenciones, entrenamiento de habilidades y tratamiento conductual) y la mitad de ellos además realizaban el IPT.

Los resultados muestran que los pacientes que habían recibido el tratamiento cognitivo (IPT) presentaban mejoras significativas en algunos dominios cognitivos. Estas mejoras a nivel cognitivo no se dieron en los sujetos que habían participado en el programa de rehabilitación global sin tratamiento cognitivo.

Las funciones cognitivas en las que se mejora el rendimiento son: el procesamiento atencional temprano, la memoria y la función ejecutiva. No obteniendo un mayor rendimiento en el tiempo de reacción y la atención continuada. Se confirman así las 
mejoras cognitivas ya detectadas en los otros estudios, aúnque el rendimiento en los tests sigue siendo inferior al presentado por sujetos normales. Además, los autores consideran que hay escasa evidencia de los cambios significativos en funciones cognitivas más globales y su generalización a un nivel superior de competencias.

\section{B. Programa Ejecutivo Frontal}

Este programa, de Morice y Delahunty(62) fue diseñado para modificar el déficit en la función ejecutiva. Está compuesto por dos módulos en los que se trabajan las habilidades de planificación y la flexibilidad cognitiva.

Se han realizado pocos estudios dirigidos a evaluar su eficacia. Los autores del programa llevan a cabo una investigación en la que obtienen que los sujetos que participan en el programa presentan, al finalizar éste, un aumento en el rendimiento en las funciones frontales y ejecutivas(44).

Estos hallazgos fueron replicados por Wykes y col.(63) en un estudio en el que compararon el programa (aplicado a 17 sujetos) con una terapia ocupacional (aplicada a 16 sujetos). Los resultados obtenidos muestran que el Programa Ejecutivo Frontal mejora el déficit en la función ejecutiva, en concreto: la flexibilidad cognitiva, la memoria de trabajo y la planificación. A los 6 meses de seguimiento los pacientes presentaban mejor funcionamiento cognitivo y autoestima que el grupo de terapia ocupacional, no difiriendo con relación a los síntomas y el funcionamiento social.

\section{Terapia de Mejora Cognitiva (CET)}

Este programa de rehabilitación psicosocial diseñado por Hogarty y Flesher(64) para pacientes ambulatorios se dirige tanto a los déficits cognitivos no sociales como sociales. Está basada en la teoría del neurodesarrollo para la rehabilitación de los pacientes esquizofrénicos(65), e intenta facilitar la cognición social abstracta mediante la selección de procesos cognitivos que subyacen a la capacidad para adquirir la cognición social adulta.

Todavía no se han realizado estudios de eficacia. Pero por el momento los autores han ofrecido resultados preliminares alentadores, observando una mejoría, al año de tratamiento, en todos los tests cognitivos, así como en las evaluaciones de cognición social, discapacidad y autoestima. Aún no disponemos de los datos de seguimiento a dos años.

\section{Conclusiones}

Una vez realizada la revisión de la eficacia de la rehabilitación cognitiva en los pacientes esquizofrénicos, podemos afirmar que hay evidencia científica con relación a: la existencia de déficits cognitivos en las personas con esquizofrenia, los ámbitos de repercusión de estos déficits (cognitivo, social, laboral...) y la posibilidad de modificar estos déficits a través de la rehabilitación cognitiva. 
Sin embargo, hay escasa evidencia en lo que atañe a la magnitud del efecto terapéutico y el tiempo de mantenimiento de los cambios, así como a la generalización de estos cambios a un nivel superior de funcionamiento, ya que los estudios de asociación entre la mejora en el funcionamiento cognitivo y el funcionamiento adaptativo no ofrecen resultados concluyentes.

Para solucionar estas inconsistencias en los distintos hallazgos y mejorar el conocimiento del efecto de la rehabilitación cognitiva en los pacientes esquizofrénicos es necesario principalmente: una mayor investigación sistemática con estudios controlados y un afianzamiento de las teorías integradoras.

Finalmente, los resultados inconsistentes con relación a la generalización de los cambios cognitivos a un nivel conductual y social, plantean la necesidad de una intervención multimodal como tratamiento adecuado de la cognición social. Es decir, programas de rehabilitación en los que se intervenga tanto en las estrategias conductuales y personales como en los déficits de procesamiento de la información.

\section{REFERENCIAS BIBLIOGRÁFICAS}

1. Heaton RK; Crowley TJ. «Effects of psychiatry disorders and their somatic treatments on neuropsychological test results», en Filskov SB y otros (eds), Handbook of Clinical Neuropsychology, New York, John Wiley, 1981:481-525.

2. Seidman LJ. «Schizophrenia and brain dysfunction: An integration of recent neurodiagnostic findings». Psychological bulletin 1983; 94:195-238.

3. Kolb B; Whishaw IQ. «Performance of schizophrenic patients on tests sensitive to left or right temporal or parietal function in neurological patients». Journal of Nervous and Mental Disease 1983;171:435-443.

4. Hoff A y otros. «Anomalous lateral sulcus asymmetry and cognitive function in firstepisode schizophrenia». Schizophr Bull 1992; 18:257-270.

5. Mohamed S y otros. «Generalized cognitive deficits in schizophrenia.A study of first episody patients». Arch Gen Psychiatry 1999; 56:749-754.

6. Heaton R y otros. «Neuropsychological deficits in schizophrenics. Relationship to age, chronicity and dementia». Arch Gen Psychiatry 1994; 51(6):469-76.

7. Bilder RM y otros. «Intellectual deficits in first-episode:evidence for progressive deterioration». Schizophr Bull 1992; 18:437-448.

8. Nuechterlein $\mathrm{KH}$ y otros. «Developmental processes in schizophrenic disorders:longitudinal studies of vulnerability and stress». Schizophr Bull 1992; 18(3):387-425.

9. Rund BR. «A review of longitudinal studies of cognitive functions in schizophrenia patients». Schizophr Bulletin 1998; 24 (3):425-435. 
10. Goldberg TE y otros. «Course of schizophrenia: neuropsychological evidence for a static encephalopathy». Schizophr Bull 1993; 19(4):797-804.

11. Andreasen NC; Olsen S. «Negative vs positive schizophrenia». Arch Gen Psychiatry 1982; 39:789-794.

12. Frith CD. The cognitive neuropsychology of schizophrenia. Eerlbaum (Reino Unido): Taylor \& Francis 1992.

13. Kremen WS. «Intellicence quotient and neuropsychological profiles in patients with schizophrenia and in normal volunteers». Biol Psychiatry 2001; 50(6):453-62.

14. Goldman R; Selemon. «Functional and anatomical aspects on prefrontal pathology in schizophrenia». Schizophr Bull 1997; 23(3):437-58.

15. Rund BR; Borg EN. «Cognitive deficits and cognitive training in schizophrenic patients:a review». Acta Psychiatr Scand 1999; 100:85-95.

16. Aleman A. y otros. «Memory impairment in schizophrenia:A meta-analysis». American Journal of Psychiatry 1999;156:1358-1366.

17. Morice R; Delahunty A. «Frontal/Executive impairments in schizophrenia». Schizophr Bulletin 1996; 22(1):125-37.

18. Green M; Walker E. «Attentional performance in positive and negative-symptom schizophrenia». J nerv ment dis 1986;174(4):208-13.

19. Deribigbe YA; Gureje O. «Symptom dimensions of schizophrenia: a selective integration of neurophysiological and neuropsychological correlates». Neuropsychobiology 1996;34(4):192-200.

20. Braff DL y otros. «The generalised pattern of neuropsychological deficits in outpatients with chronic schizophrenia with heterogeneous Wisconsin card sorting test results». Archives of General Psychiatry 1991;48:891-898.

21. Kischka U; Spitzer M; Kammer T. «Frontal-subcortical neuronal circuits». Fortschr neurol psychiatr 1997 ; 65(5):221-31.

22. Rubin P y otros. «Altered modulation of prefrontal and subcortical brain activity in newly diagnosed schizophrenia and schizophreniform psychosis: a regional cerebral blood flow study». Archives of General Psychiatry 1991; 48:987-995.

23. Dolan RJ y otros. «Abnormal neural integration related to cognition in schizophrenia». Acta Psychiatr Scand 1999; 395(99):51-57.

24. Spaulding W y otros. «Cognitive functioning in schizophrenia:Implications for psychiatric rehabilitation». Schizophrenia Bulletin 1999; 25(2):275-289.

25. Allen HA. «Cognitive processing and its relationship to symptoms and social functioning in schizophrenia». British Journal of Psychiatry 1990; 156:201-203. 24.

26. Brekke J; Raine A; Ansel M. «Neuropsicological and psychophysiological correlates of psychosocial functioning in schizophrenia». Schizophrenia Bulletin 1997; 23:19-28.

27. Dickerson F y otros. «Neurocognitive deficits and social functioning and in otupatients with schizophrenia». Schizophrenia Res 1996; 21(2):75-83. 
INFORMES

28. Dickerson F y otros. «Social functioning and neurocognitive deficits in otupatients with schizophrenia: a 2-year follow-up». Schiz Res 1999 37(1):13-20.

29. Smith T.E y otros. «The relative influences of symptoms, insight and neurocognition on social adjustment in schizofrenia and schizoaffective disorder». J Nerv Ment Dis 1999; 187(2):102-8.

30. Green M. «What are the functional consequences of neurocognitive deficits in schizofrenia? Am J Psychiatry 1996; 153(3):321-330.

31. Goldman R. «Neuropsychological prediction of treatment efficacy and one-year outcome in schizophrenia». Psychopatology 1993; 26:122-126.

32. Silverstein ML; Harroe M; Bryson GJ. «Neuropsychological prognosis and clinical recovery». Psychiatry Res 1994; 52(3):265-72.

33. Penadés R y otros. «Deficit in schizophrenia:Relationship between negative simptoms and neurocognition». Compr Psychiatry 2001; 42(1):64-69.

34. Kern RS; Green MF; Satz P. «Neuropsychological predictors of skills training for chronic psychiatric patients. Psychiatry Res 1992; 43(3):223-30.

35. Smith TE y otros. «Symptoms and neurocognition as rate limiters in skills Training for psychotic patients». American Journal of Psychiatry 1999; 156:1817-1818.

36. Mueser K; Bond G; Drake R. «Community-Based treatment of schizophrenia and other severe mental disorders:Treatment Outcomes?». Medscape Mental Health 2001; 6(1).

37. Goldsetein G; Beers SR; Shemnasky WJ. «Neuropsychological diferences between schizophrenic patients with heterogeneous Wisconsin Card Sorting Test performance». Schizophrenic Research 1996; 21(1):13-8.

38. Goldberg TE y otros. «Further evidence for dementia of prefrontal type in schizophrenia? A controlled study of teaching the Wisconsin card sorting test». Archives of General Psychiatry 1987; 44:1008-1014.

39. Green M y otros. «Wisconsin Card Sorting Test Performance in schizophrenia: Remediation of stubborn deficit». Am J Psychiatry 1992 149:62-67.

40. Bellack A y otros. «Remediation of cognitive deficits in schizophrenia». Am J Psychiatry 1990; 147(12):1650-1655.

41. Tompkins L; Goldman R; Axelrod B. «Modifyng the WCST performance of schizophrenic patients». Am J Psychiatry 1991; 148(11):1613.

42. Green MF y otros. «Teaching the Wisconsin Card Sort to schizophrenic patients. Archives of General Psychiatry 1990; 47:91-92.

43. Hellman SG y otros. «Monetary reinforcement and Wisconsin Card Sorting performance in schizophrenia: why show me the money?». Schizophrenia Research 1998; 34(1-2):67-75.

44. Delahunty A; Morice R; Frost B. «Specific cognitive flexibility rehabilitation in schizophrenia». Psychol Med 1993; 23(1):221-7.

45. Delahunty A; Morice R. «Rehabilitation of frontal/executive impairments in schizophrenia». Aust N Z J Psychiatry 1996; 30(6):760-7. 
46. Metz $\mathrm{J}$ y otros. «Maintenance of training effects on the wisconsin card sorting test by patients with schizophrenia or affective disorders». Am J Psychiatry 1994; 151:120-122.

47. Young DA; Freyslinger MG. «Scaffolded instruction and the remediation of Wisconsin Card Sorting Test deficits in chronic schizophrenia». Schizophrenic Research 1995; 16(3):199-207.

48. Kern RS y otros. «A training procedure for remediating WCST deficits in chronic psychotic patients: an adaptation of errorless learning principles». J Psychiatr Res 1996; 30(4):283-94.

49. Bellack AS y otros. «Generalization effects of training on the Wisconsin Card Sorting Test for schizophrenia patients». Schizophr Res 1996; 19(2-3):189-94.

50. Benedict $\mathrm{R}$ y otros. «Effects of attention training on information processing in schizophrenia». Schizophr Bull 1994; 20(3):537-46.

51. Suslow T; Schonauer K; Arolt V. «Attention training in the cognitive rehabilitation of schizophrenic patients:a review of efficacy studies». Acta Psichiatr Scand 2001; 103:15-23.

52. Koh SD; Peterson RA. «Perceptual memory for numerousness in nonpsychotic schizophrenics». J abnorm psychol 1974; 83(3):215-26.

53. Koh SD; Kayton L. «Memorization of «unrelated» word strings by young nonpsychotic schizophrenics». J abnorm psychol 1974;83(1):14-22.

54. Wexler B y otros. «Preliminary evidence of improved verbal working memory performance and normalitzation of task-related frontal lobe activation in schizophrenia following cognititve exercices». Am J Psychiatry 2000; 157(10):1694-1697.

55. Wykes T. «What are we changing with neurocognitive rehabilitation? Illustrations from two single cases of changes in neuropsychological performance and brain systems as measured by SPECT». Schizophr Res 1998; 34(1-2):77-86.

56. Wykes $\mathrm{T}$ y otros. «Effects on the brain of a psychological treatment: cognitive remediation therapy: functional magnetic resonance imaging in schizophrenia». $\mathrm{Br} \mathrm{J}$ Psychiatry 2002; 181:144-52.

57. Brenner H; Pfammatter M. «Psychological therapy in schizophrenia:what is the evidence?» Acta Psychiatr Scand 2000; 102 (supl.407):74-77.

58. Brenner $\mathrm{H}$ y otros. Integrated psychological therapy for schizophrenic patients. Bern:Hogrefe \& Huber 1995.

59. Zubin J; Spring B. «Vulnerability. A new view of schizophrenia». J Abnormal Psychology 1977; 86(2):103-26.

60. Brenner H y otros . «Treatment Of Cognitive Dysfunctions And Behavioral Deficits In Schizophrenia». Schizophr Bull 1992; 18(1):21-6.

61. Spaulding W y otros. «Effects of cognitive treatment in psychiatric rehabilitation». Schizophr Bull 1999; 25(4):657-76.

62. Morice R; Delahunty A. Treatment strategies for the remediation of neurocognitive dysfunction in schizophrenia. En: Pantelis C, Nelson HE, Barnes TR (eds), 1996 pp.447459. 
INFORMES

63. Wykes $\mathrm{T}$ y otros. «The effects of neurocognitive remediation on executive processing in patients with schizophrenia». Schizophr Bull 1999; 25(2):291-307.

64. Hogarty G; Flesher S. «Practice principles of cognitive enhancement therapy for schizophrenia. Schizophrenia». Schizophr Bull 1999; 25(4):693-708.

65. Hogarty G; Flesher S. «Developmental theory for a cognitive enhancement therapy of schizophrenia». Schizophr Bull 1999; 25(4):677-92.

\footnotetext{
* Residente de Psicología Clínica. Institut d'Assistència Sanitaria (Girona)

** Residente de Psiquiatría. Institut d'Assistència Sanitaria (Girona)

*** Residente de Psicología Clínica. Institut d'Assistència Sanitaria (Girona)

**** Residente de Psiquiatría. Institut d'Assistència Sanitaria (Girona)

***** Residente de Psiquiatría. Institut d'Assistència Sanitaria (Girona)
} 